\title{
New occurrence of B chromosomes in Partamona helleri (Friese, 1900) (Hymenoptera, Meliponini)
}

\author{
Cinthia Caroline Cardoso Martins ${ }^{1}$, Olivia Maria Pereira Duarte ${ }^{1}$, Ana Maria Waldschmidt ${ }^{2}$, \\ Rogério Marco de Oliveira Alves ${ }^{3}$ and Marco Antônio Costa ${ }^{1}$ \\ ${ }^{1}$ Universidade Estadual de Santa Cruz, Departamento de Ciências Biológicas, Ilhéus, BA, Brazil. \\ ${ }^{2}$ Departamento de Ciências Biológicas, Universidade Estadual do Sudoeste da Bahia, Jequié, BA, Brazil. \\ ${ }^{3}$ Universidade Federal do Recôncavo Baiano, Cruz das Almas, Bahia, Brazil.
}

\begin{abstract}
Cytogenetic analyses of the stingless bee Partamona hellericollected in the state of Bahia, Northeast Brazil revealed the chromosome numbers $n=18$ in the haploid males and $2 n=35$ in the diploid females. All karyotypes displayed one large acrocentric $B$ chromosome, which differs from the minute $B$ chromosomes previously described in the populations from southeastern Brazil. Giemsa staining, C-banding and DAPI/CMA fluorochrome staining also revealed a remarkable interpopulational divergence regarding both the regular karyotype and the $\mathrm{B}$ chromosomes. The $\mathrm{B}$ chromosomes found in the samples from Jequié, Bahia, were entirely heterochromatic, while those found in Cravolândia, Bahia, displayed a euchromatic portion at the telomeric end of the long arm. $\mathrm{CMA}_{3}$ labeling sites varied from seven to eight between the two localities in Bahia, due to the presence of an extra GC-rich block in the karyotype of the samples from Jequié. This is the first report of a large B chromosome in $P$. helleriand reveals the occurrence of a geographic differentiation within this species.
\end{abstract}

Key words: stingless Bee, Partamona helleri, geographic variation, supernumerary chromosomes.

Received: October 7, 2008; Accepted: March 11, 2009.

Partamona is a Neotropical genus of stingless bees with an ample distribution in a wide variety of habitats going from southern Brazil to central Mexico. These habitats include rain forests, cerrado (Brazilian savanna), caatinga, and highlands. Given the existence of morphologically similar species, this group has a problematic taxonomy. Some species can only be recognized by their nesting behavior or nest entrance architecture (Pedro and Camargo, 2003).

Of the 33 species currently recognized (Pedro and Camargo, 2003), only eight, P. pearsoni (Tarelho ZVS, 1973, MSc Dissertation, University of São Paulo), $P$. seridoensis (Brito-Ribon et al., 1999, 2005;), P. aiylae, P. vicina, P. mulata, P. nhambiquara (Brito-Ribon et al., 1999), P. peckolti (Brito et al., 2003), and P. helleri (Costa et al., 1992; Brito et al., 1997, 2005), were cytogenetically studied. All these species showed the regular chromosome number $2 \mathrm{n}=34$, but $P$. helleri showed a diploid numeric variation ranging from $2 n=34$ to $2 n=38$, due to the occurrence of up to four minute $\mathrm{B}$ chromosomes per individual (Costa et al., 1992; Brito et al., 1997, 2005; Tosta et al., 2004).

Send correspondence to Marco Antônio Costa. Departamento de Ciências Biológicas, Universidade Estadual de Santa Cruz, 45662-000 Ilhéus, BA, Brazil. E-mail: costama@uesc.br.
B chromosomes are extra chromosomes to the regular complement and are characterized by their dispensability, independent evolution, and non-Mendelian patterns of inheritance (Beukeboom, 1994). Their origin is a matter of recurrent debate among cytologists. Some have proposed that the appearance of the B chromosomes suggests the involvement of rearrangements in the regular chromosomes, e.g., centric fragment formation through chromosome fusions (Camacho et al., 2000). An alternative hypothesis suggests an origin through interspecific hybridization as observed in the fish Poecilia formosa (Schartl et al., 1995) and in the wasp Nasonia vitripennis (McAllister and Werren, 1997).

Brito et al. (1997) distinguished two morphological types of B chromosomes occurring in P. helleri in southeastern Brazil. Although the presence of the minute B's was common in the previously studied population, the fourfold B dosage $[2 \mathrm{n}=38$ chromosomes, Tosta et al. (2004)] was rare. This finding suggests the existence of a mechanism controlling or preventing the accumulation of B chromosomes in individuals.

Recently molecular studies have been started, in an attempt to better understand the population dynamics of $\mathrm{B}$ chromosomes in P. helleri. Tosta et al. (2007) developed a SCAR (Sequence Characterized Amplified Region) marker 
potentially useful for analyzing the frequency, geographic distribution, transmission, or effects of the $\mathrm{B}$ chromosome in the adult organism. However, despite the contributions given by previous studies, the origin of the B's in P. helleri is so far still unclear.

Here, we report a new type of $\mathrm{B}$ chromosome in populations from the northern limits of the distribution of $P$. helleri in the state of Bahia, Brazil. Differences from previous descriptions found in the regular karyotype are also discussed.

One nest of $P$. helleri from Jequié $\left(13^{\circ} 52^{\prime} \mathrm{S}\right.$, $\left.40^{\circ} 13^{\prime} \mathrm{W}\right)$ and one nest from Cravolândia $\left(13^{\circ} 21^{\prime} \mathrm{S}\right.$, $39^{\circ} 48^{\prime} \mathrm{W}$ ), both in the state of Bahia, Northeast Brazil, were collected for cytogenetic analyses.

Metaphases were obtained from cerebral ganglia of prepupae, following the protocol described by Imai et al. (1988). A total of 50 specimens were analyzed.

We performed $\mathrm{C}$ banding according to Sumner (1972), with the slight modifications proposed by Pompolo and Takahashi (1990). DAPI/CMA 3 staining was done as described by Schweizer (1980).

C-banded and conventional Giemsa-stained chromosomes were analyzed and photographed with a Leica DLMS photomicroscope using HQ film. Fluorescent images were captured in a Leica DMRA2 photomicroscope using the IM50 software. In order to compare our results with previous studies, the karyotypes were arranged by decreasing order of length of euchromatic arms, and the chromosome nomenclature followed Imai (1991), with metacentric chromosomes showing pericentomeric $\left(\mathrm{M}^{\mathrm{cc}}\right)$, centromeric $\left(\mathrm{M}^{\mathrm{c}}\right)$, pericentromeric and telomeric $\left(\mathrm{M}^{\mathrm{cct}}\right)$, centromeric and telomeric $\left(\mathrm{M}^{\mathrm{ct}}\right)$, and pericentromeric and interstitial $\left(\mathrm{M}^{\mathrm{cci}}\right)$ heterochromatic bands (see the original reference for more details).

In the present analyses, the karyotype of $P$. helleri showed 35 chromosomes in the females and 18 chromosomes in the males. For the Cravolândia samples, the Cbanded diploid karyotypic formula found was $2 \mathrm{k}=28 \mathrm{M}^{\mathrm{cc}}+$ $4 \mathrm{M}^{\mathrm{c}}+2 \mathrm{M}^{\mathrm{cct}}+1 \mathrm{~B}$ (Figure 1d).

Previous studies had reported the karyotypic formula $2 \mathrm{k}=20 \mathrm{M}^{\mathrm{cc}}+4 \mathrm{M}+8 \mathrm{M}^{\mathrm{ct}}+2 \mathrm{M}^{\mathrm{cci}}+1 \mathrm{~B}$ for the $2 \mathrm{n}=35$ karyotypes sampled in the state of Minas Gerais (Brito et al., 1997, 2005). In our study, metacentric chromosomes with pericentromeric and interstitial heterochromatin $\left(\mathrm{M}^{\mathrm{cci}}\right)$ were not observed.

The novel B chromosomes found in the Bahia samples were much larger than those described in the population from southeastern Brazil (Brito et al., 1997, 2005). They had an acrocentric morphology and a size comparable to the chromosomes of the regular complement. The B chromosomes observed in the Jequié samples were entirely heterochromatic, whereas those found in Cravolândia showed a euchromatic portion at the telomeric end of the long arm (Figures 1c, d). These results reveal a substantial karyotypic divergence among the populations studied so

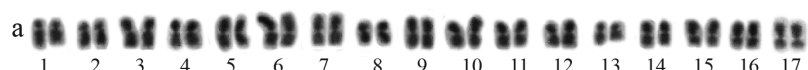

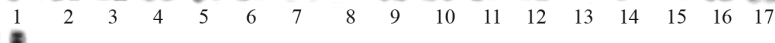
8

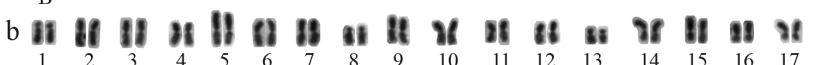
B

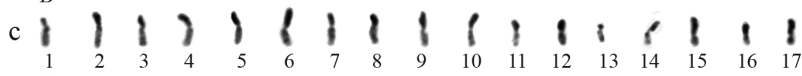
I

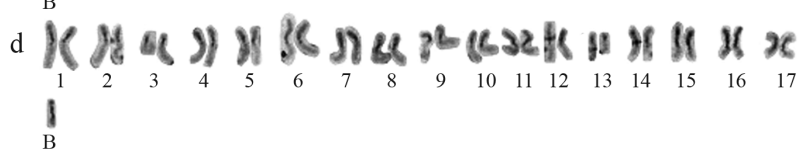

Figure 1 - $P$. helleri karyotypes with one B chromosome of: a) a female $(2 \mathrm{n}=35)$ from Jequié, b) a female $(2 \mathrm{n}=35)$ from Cravolândia after Giemsa staining, c) a male $(n=18)$ from Jequié, and d) a female $(2 n=35)$ from Cravolândia after C-banding.

far, due to structural changes in both the regular and the B chromosomes.

Brito et al. (1997) classified the minute B's found in the southeastern Brazilian populations as B1 (heterochromatic submetacentrics) and B2 (acrocentrics, with C-banding undetermined due to their small size). Partially and entirely heterochromatic B chromosomes were found in another bee species, Melipona quinquefasciata (Marla P. Rocha, personal communication).

The DAPI fluorochrome stained the heterochromatin in all centromeric and pericentromeric regions of regular chromosomes (Figures $2 \mathrm{~b}, \mathrm{c}$ ), but the $\mathrm{CMA}_{3}$ fluorochrome staining was variable. Specimens from Jequié showed eight $\mathrm{CMA}_{3}^{+}$labeling sites, seven on the regular chromosomes and one on the $\mathrm{B}$ chromosome (Figure 2a). In the samples from Cravolândia, only seven $\mathrm{CMA}_{3}$ labeling sites were observed, six on the regular chromosomes and one on the $\mathrm{B}$ chromosome (Figures 2c). The latter result agrees with Brito et al. (2005), who also found seven $\mathrm{CMA}_{3}{ }^{+}$markings on regular chromosomes and one on the minute $\mathrm{B}$ in $P$. helleri from southeastern Brazil. These authors also found a heteromorphism in the second chromosome pair due to a difference in the size of the heterochromatic/ $\mathrm{CMA}_{3}{ }^{+}$long arm, a result not observed in the Bahia populations. Similar heterogeneous AT- and GC-rich heterochromatin blocks were observed in other meliponine species, such as Plebeia sp. and Melipona sp. (Maffei et al., 2001), and Partamona peckolti (Brito et al., 2003).

The present analysis using different techniques revealed the occurrence of a geographic differentiation between the populations of $P$. helleri. We observed karyotypic differences in the size, morphology, and distribution of the heterochromatin in the chromosomes of the regular complement and of the B chromosomes. Our results show that the $\mathrm{B}$ chromosome system of $P$. helleri is more complex than previously assumed. The large B chromosomes found in the Brazilian Northeast region can be an important element for future evolutionary studies of this species. 

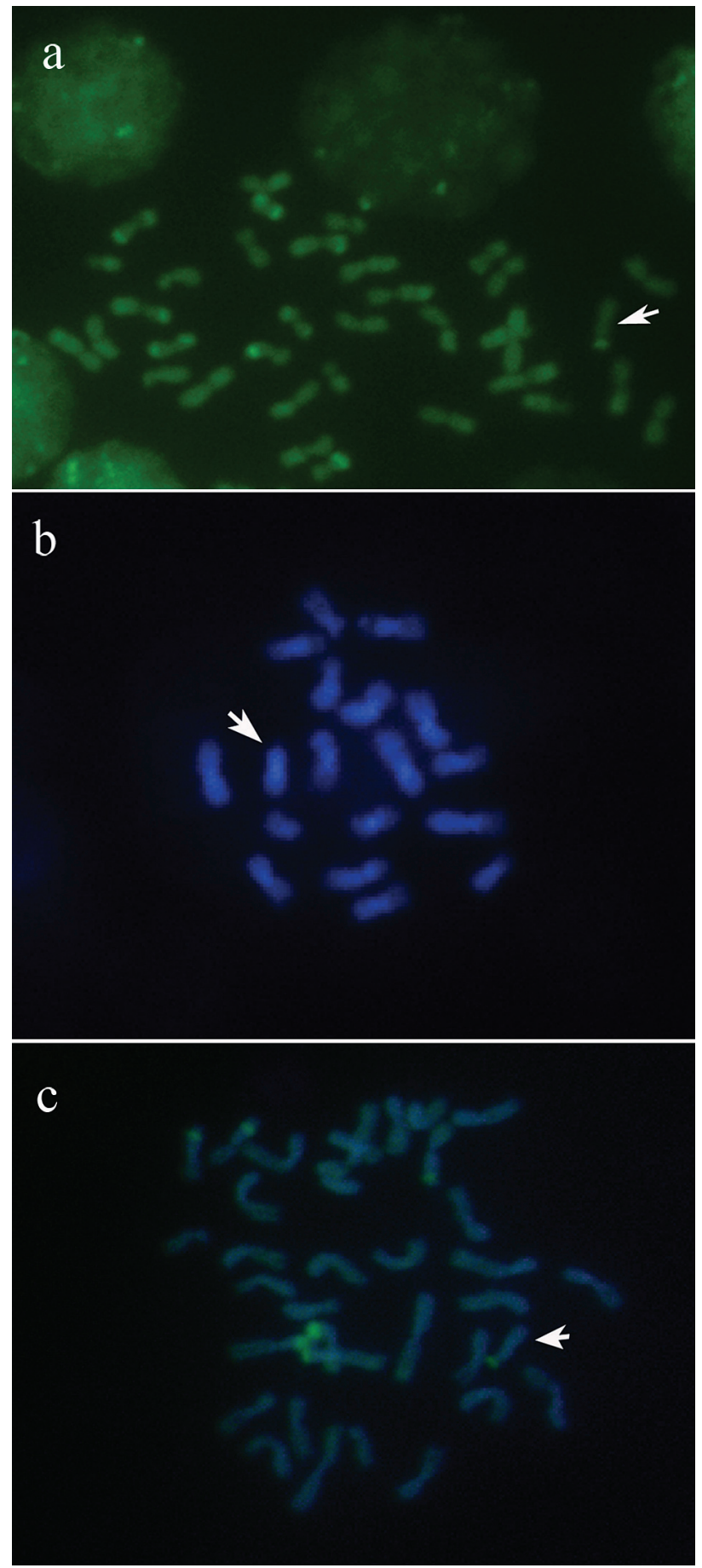

Figure 2 - Metaphases of $P$. helleri with one B chromosome of: a) $\mathrm{CMA}_{3}$-stained female karyotype from Jequié $(2 \mathrm{n}=35)$; b) a DAPI-stained male karyotype from Jequié $(\mathrm{n}=18)$; and c) a DAPI/ $\mathrm{CMA}_{3}$-sequential stained female karyotype $(2 \mathrm{n}=35)$ from Cravolândia. The arrows indicate the B chromosomes.

In the study carried out by Tosta et al. (2007), the authors found a SCAR (Sequence Characterized Amplified Region) marker associated with the minute B chromosomes of animals from the southeastern region. This was the first attempt to use molecular data to investigate the ef- fect of B chromosomes on the individuals and showed that the approach could be effective for future population studies of this species, especially concerning the origin of the B's.

The karyotypic differences described indicate that the $\mathrm{B}$ chromosomes found in the present study and those previously reported in the southeastern region could have had distinct origins, probably involving rearrangements in several of the regular chromosomes. The so far undefined origins and patterns of the B chromosome inheritance can be clarified with an ample analysis of its variation and geographic distribution in this species. Other approaches, including molecular and cytogenetic data, will help to clarify the cytotaxonomy and the mechanisms involved in the karyotype evolution of $P$. helleri. We have already been carrying out new samplings in order to further characterize the cytogenetic and molecular diversity of this species, especially in Bahia where the large Bs were found.

\section{Acknowledgments}

We thank Dr. Silvia Regina de Menezes Pedro from Universidade de São Paulo, Ribeirão Preto, for the identification of the species and Dr. Janisete G. Silva-Miller for the helpful comments on the manuscript. This research was supported by FAPESB (Fundação de Amparo à Pesquisa do Estado da Bahia).

\section{References}

Beukeboom LW (1994) Bewildering Bs: An impression of the 1st B-chromosome conference. Heredity 73:328-336.

Brito RM, Costa MA and Pompolo SG (1997) Characterization and distribution of supernumerary chromosomes in 23 colonies of Partamona helleri (Hymenoptera, Apidae, Meliponinae). Braz J Genet 20:185-188.

Brito-Ribon RM, Miyazawa CS and Pompolo SG (1999) First karyotype characterization of Four Species of Partamona (Friese, 1980) (Hymenoptera, Apidae, Meliponini) in Mato Grosso State - Brazil. Cytobios 100:19-26.

Brito RM, Caixeiro APA, Pompolo SG and Azevedo GG (2003) Cytogenetic data of Partamona peckolti (Hymenoptera, Apidae, Meliponini) by $\mathrm{C}$ banding and fluorochrome staining with $\mathrm{DA} / \mathrm{CMA}_{3}$ and DA/DAPI. Genet Mol Biol 26:5357.

Brito RM, Pompolo SG, Magalhães MFM, Barros EG and Sakamoto-Hojo ET (2005) Cytogenetic characterization of two Partamona species (Hymenoptera, Apinae, Meliponini) by fluorochrome staining and localization of $18 \mathrm{~S}$ rDNA clusters by FISH. Cytologia 70:373-380.

Camacho JPM, Sharbel TF and Beukeboom LW (2000) B-chromosome evolution. Phil Trans R Soc Lond B 355:163-178.

Costa MA, Pompolo SG and Campos LAO (1992) Supernumerary chromosomes in Partamona cupira (Hymenoptera, Apidae, Meliponinae). Rev Bras Genet 15:801-806.

Imai HT (1991) Mutability of constitutive heterochromatin (Cbands) during eukaryotic chromosomal evolution and their cytological meaning. Jpn J Genet 66:653-66. 
Imai HT, Taylor RW, Crosland MWJ and Crozier RH (1988) Modes of spontaneous chromosomal mutation and karyotype evolution in ants with reference to the minimum interaction hypothesis. Jpn J Genet 63:159-185.

Maffei EM, Pompolo SG, Silva-Junior JC, Caixeiro AP, Rocha MP and Dergam JA (2001) Silver staining of nucleolar organizer regions (NOR) in some species of Hymenoptera (bees and parasitic wasps) and Coleoptera (lady-beetle). Cytobios 104:119-125.

McAllister BF and Werren JH (1997) Hybrid origin of a B chromosome (PSR) in the parasitic wasp Nasonia vitripennis. Insect Mol Biol 4:253-262.

Pedro SEM and Camargo JMF (2003) Meliponini neotropicais: O gênero Partamona Schwarz, 1939 (Hymenoptera, Apidae). Rev Bras Entomol 47:1-117.

Pompolo SG and Takahashi CS (1990) Chromosome numbers and C-banding in two wasp species of the genus Polistes (Hymenoptera Polistine, Polistini). Insect Soc 37:251-257.

Schartl M, Nanda I, Schlupp I, Wilde B, Epplen JT, Schmid M and Parzefall J (1995) Incorporation of subgenomic amounts of
DNA as compensation for mutational load in a gynogenetic fish. Nature 373:68-71.

Schweizer D (1980) Simultaneous fluorescent staining of R bands and specific heterochromatic regions (DA-DAPI bands) in human chromosomes. Cytogenet Cell Genet 27:190-193.

Sumner AT (1972) A simple technique for demonstrating centromeric heterochromatin. Exp Cell Res 75:304-306.

Tosta VC, Fernades-Salomão TM, Tavares MG, Pompolo SG, Barros EG and Campos LAO (2004) A RAPD marker associated with B chromosomes in Partamona helleri (Hymenoptera, Apidae). Cytogenet Genome Res 106:279-283.

Tosta VC, Tavares MG, Fernandes-Salomão TM, Barros EG, Campos LAO and Camacho JPM (2007) Development of a SCAR marker for the analysis of B chromosome presence in Partamona helleri (Hymenoptera, Apidae). Cytogenet Genome Res 116:127-129.

\section{Associate Editor: Yatiyo Yonenaga-Yassuda}

License information: This is an open-access article distributed under the terms of the Creative Commons Attribution License, which permits unrestricted use, distribution, and reproduction in any medium, provided the original work is properly cited. 